\title{
COMERCIO INTERNACIONAL MEDITERRÁNEO EN EL SIGLO VIII A.C.
}

\author{
POR
}

\author{
PALOMA CABRERA BONET
}

Museo Arqueológico Nacional (Madrid)

\begin{abstract}
RESUMEN
El siglo vill a.C. es el momento de la recuperación, por primera vez desde el colapso del sistema a fines de la Edad del Bronce. de un verdadero comercio internacional a gran escala, cuyos protagonistas más activos serán fenicios y griegos. En este trabajo queremos analizar algunos aspectos de este fenómeno, desde el Egeo al extremo Occidente, que, a través de una vasta red de rutas comerciales, permitió una amplia interacción multinacional. Entendemos asi el comercio internacional como un factor clave en el proceso de cambio económico, social y politico de las áreas mediterráneas inmersas en este complejo "sistema-mundo".
\end{abstract}

\section{SUMMARY}

The vil century B.C. saw, for the first time after the breakdown of the system in the Late Bronze Age, the revival of a true international trade at a growing scale, especially protagonized by Phoenicians and Greeks. In this article we analyze some aspects of this phenomenon, from the Aegean to the Far West, which made possible, through a vaste network of trading routes, a large multinational interaction. We understand the international trade as a key factor in the process of economic, social and political changes of the Mediterranean areas submerged in this complex "world-system".

\section{INTRODUCCIÓN}

Estamos asistiendo en estos últimos años a la revitalización de un viejo debate, largamente sostenido y nunca olvidado, pero situado ahora en un punto crucial gracias al planteamiento de nuevos modelos teóricos y al avance producido en la investigación arqueológica con la ayuda de nuevos instrumentos de análisis: me refiero al problema de la caracterización de los sistemas económicos de las sociedades antiguas, precapitalistas, el papel del comercio y los intercambios, los mecanismos a través de los cuales se desarrollan, y sus consecuencias en el or- den social y politico, $y$, en definitiva, en el proceso histórico del cambio cultural.

Superados ya los modelos invasionistas y difusionistas de la Arqueologia tradicional historicista para explicar el cambio cultural, y con las aportaciones teóricas y metodológicas de la Arqueología sistémica (Renfrew, 1969, 1984), especialmente en lo que se refiere a la valoración de las formas de especialización e intercambio que promueven complejidad, esto es, estratificación social, integración política y especialización institucional, y del sistema de intercambio en el proceso de interacción entre los diferentes subsistemas que conduce al cambio cultural (Schortman y Urban, 1987), nos encontramos hoy ante nuevos enfoques en los que se redimensiona el papel del comercio y de los intercambios bajo una perspectiva mucho más amplia (Mc C. Ada$\mathrm{ms}, 1974)$. La valoración de los aspectos sociales, políticos y simbólicos del intercambio es el rasgo predominante de los nuevos enfoques.

Por una parte se ofrece hoy una visión más completa, subrayando lo que Melas $(1991,389)$ llama la "sociologia artefactual», que "implica la consideración de dos cuestiones dialécticamente relacionadas: el efecto de la organización socio-económica y política sobre la distribución e intercambio de artefactos, y el papel de los artefactos y su intercambio marcando esferas de interacción social».

Por otra, se subraya el valor de la demanda y del consumo en el movimiento de productos (Sombart, 1967; Sherrat, 1991, 1993; Knapp, 1993) y la importancia de los intercambios para extender el control sociopolítico de las élites a través de la influencia económica, del poder coercitivo y la legitimación, entendiendo que el poder se obtiene a través del control monopolizador sobre ciertos productos de 
prestigio (incluidos los extranjeros) que constituyen la riqueza, y que el control y manipulación de riqueza es el factor clave en la constitución del poder politico (Brumfiel y Earle. 1987).

Pero además, las nuevas perspectivas también se amplian cultural y geográficamente. En el análisis del desarrollo de las sociedades complejas en el Mediterráneo y Europa en la Edad del Bronce se entiende el comercio no sólo como un factor clave. sino también con un caracter internacional dentro de «un sistema mundial cosmopolita caracterizado por un cierto grado de integración cultural y politica» (Melas, 1991, 391). Este sistema económico y social global, que deriva del modelo de Wallerstein sobre el "sistema-mundo" (Wallerstein, 1974; Rowlands, Larsen, Kristiansen, 1987; Champion, 1989), concibe las sociedades individuales como partes interligadas de unos centros de desarrollo en el contexto mediterráneo (Rowlands, 1987). Son estos vínculos sociales y económicos existentes entre unidades politicas de la Edad del Bronce los que constituyen el nuevo punto de referencia, superando el análisis aislado, como entidades geográficas separadas, entendiendo que «el comercio estaba dialécticamente ligado a un mundo fuertemente entretejido, contribuyendo a su integración y beneficiándose de ella, a través de una interacción multinacional que operaba en una vasta red de extensas rutas comerciales» (Melas, 1991, 391).

Estos nuevos enfoques teóricos han sido aplicados al análisis del comercio y el intercambio de las sociedades precapitalistas del Próximo Oriente, Europa y Mediterráneo, sobre todo durante la Edad del Bronce y época romana, pero son escasos aún los trabajos referidos a la Edad del Hierro, y especialmente a los primeros siglos del I milenio a.C.

Para el mundo griego quizás el peso de las teorías clásicas weberianas, especialmente de Hasebroek (1965) y Finley (1973), hayan retrasado la entrada de nuevos análisis renovadores.

Dentro de las nuevas corrientes teóricas, la aproximación más reciente, y más completa, con una base arqueológica, es la de S.y A.Sherrat (1993), que nos ofrece además una visión de conjunto más amplia, trascendiendo el marco del Egeo, para integrar el comercio griego en el crecimiento económico de todo el Mediterráneo en los primeros siglos del I milenio.

Para los Sherrat, una de las fases más cruciales en el desarrollo de la economía mediterránea es la transición de la Edad del Bronce a la del Hierro. Durante este periodo de transformación social fundamental, las economías dominantes del segundo milenio dieron paso a formas menos centralizadas de organización económica que caracterizaron un nuevo periodo de crecimiento acelerado que duró todo el primer milenio.

El nuevo modelo empezó a tomar forma en los siglos anteriores al 1000 a.C. en el proceso que marca la desaparición de los centros palaciales de la Edad del Bronce y las nuevas fuerzas sociales que fueron liberadas por su disolución. En este nuevo modelo fue fundamental la disolución del control estatal del comercio de productos de lujo, y la creación de un marco económico nuevo. En respuesta a la erosión del control directo de la actividad económica surgieron nuevas formas de poder político, en las que la definición territorial cobró una importancia vital, tanto a nivel de las nuevas ciudades estado, como de los nuevos imperios basados en el control politico-militar. Como en la Edad del Bronce, las estaciones comerciales de ultramar relacionadas con la adquisición de materias primas se establecieron en áreas menos desarrolladas, en la periferia del mundo urbano, a lo largo de todo el Mediterráneo. Los requerimientos de tributos, el comercio y el gasto militar derivado de los conflictos surgidos por la definición territorial, incrementaron la necesidad de remanentes en lingotes, que fueron adquiridos por la exploración de ultramar y la intensificación de las empresas mineras. Si, en palabras de los Sherrat $(1993,374)$, wen el 1000 a.C. gran parte del Mediterráneo era efectivamente prehistórico, hacia el 500 a.C. estaba formado por una serie de zonas bien diferenciadas dentro de un sistema-mundo. La transformación no se debió a la difusión pasiva de la 'civilización', sino a una intervención y respuesta activas», $\mathrm{y}$ "ni difusión ni autonomía pueden describir adecuadamente la naturaleza de este proceso de desarrollo, sino una co-evolución dentro de los límites, cada vez más amplios, y la especialización de un creciente sistema-mundo"».

Es en este marco teórico donde pretendemos analizar diversos aspectos del comercio internacional mediterráneo durante el siglo viII y sus consecuencias económicas, sociales y políticas en determinados ámbitos, y muy especialmente en la península ibérica.

\section{EL DESARROLLO DEL COMERCIO INTERNACIONAL MEDITERRÁNEO EN EL SIGLO VIII A.C.: EL EGEO}

El siglo VIII es el siglo que asiste al gran despegue económico de Grecia, a la consolidación de nuevas estructuras sociales y politicas, y especialmente de la polis, a su expansión demográfica, a la consolidación de las relaciones interegionales, a la 
gran expansión comercial y al comienzo de la expansión colonial. Es el momento en el que también asistimos a la consolidación de determinadas expresiones simbólicas, engarzadas intimamente en la estructura social, como son la consolidación de un lenguaje artistico geométrico, la consolidación de unos rituales y la monumentalización de los espacios destinados al ritual, el surgimiento de la escritura griega y la composición de los Poemas Homéricos, elementos todos ellos vehiculos de manifestaciones ideológicas de valor y estatus, $y$ factores de cohesión al servicio de las estructuras de dominio.

Creemos que uno de los factores clave en este proceso de desarrollo económico, social y politico fue la inmersión de las ciudades griegas en el comercio internacional mediterráneo a partir del siglo IX, pero especialmente en el siglo VIII. El análisis de determinados fenómenos socio-económicos, intimamente relacionados, asi nos lo confirman: 1) la intensificación de la producción, y la especialización de la misma, pensada ya no en la subsistencia o en la simple redistribución intraregional, sino en el intercambio a larga distancia; 2 ) la búsqueda de nuevos mercados y la creación de establecimientos comerciales en el Mediterráneo central; 3 ) la aceleración del proceso de consolidación del predominio de determinados grupos sociales que controlan los medios de producción y/o el aprovisionamiento de productos de valor y su redistribución, y de grupos sociales que controlan las redes de intercambio. El proceso no se desarrolló de la misma forma en todas las poleis, ni a la misma escala. Eubea y Corinto serán las grandes protagonistas de la expansión económica griega, precisamente por ser las grandes protagonistas griegas del comercio internacional. Atenas, pionera de la expansión comercial en el siglo ıx, tendrá en el siglo vıIı un desarrollo más vinculado con la explotación de los recursos del territorio, pero no será ajena al comercio y a la generación de riqueza que éste promovió.

El siglo Ix habia asistido al incremento de las comunicaciones marítimas dentro del Egeo y de los intercambios con el Próximo Oriente. El estimulo de los intercambios a larga distancia y la incipiente inmersión en el comercio internacional de determinados centros - Atenas y Lefkandi especialmentehabian promovido un inicial desarrollo económico. Pero es a partir de comienzos del siglo viI, del Gemétrico Medio II, cuando se consolida este proceso expansionista. En este momento las costas del Atica comienzan a repoblarse, hay una vigorosa actividad marítima, un aumento en los niveles de producción cerámica, cuyos productos se extienden y alcanzan a casi todos los centros del mundo griego, contribuyendo a crear una koine estilistica en el Egeo. Durante esta época las producciones ceramicas áticas llegan al Levante: a Chipre y a la costa Siria. Es el momento también de la expansión corintia hacia Itaca, hacia el camino occidental. Eubea, por su parte. poco después del 800 está realizando sus primeros movimientos hacia las costas occidentales de Italia. La expansión griega hacia la búsqueda de mercados atractivos donde conseguir no sólo materias primas, especialmente metales, sino también objetos de valor, productos de lujo, lleva a comerciantes griegos. eubeos y cicládicos especialmente, a establecer pequenoos asentamientos en las costas levantinas, caso de Al Mina. Tell Sukas o Ras el-Bassit. Por su parte, las importaciones orientales, que habian aparecido en Grecia durante el siglo $1 \mathrm{x}$ - pateras fenicias de bronce, objetos de fayenza, pasta vitrea y marfil, escarabeos, etc. - y que a comienzos del viI habian sufrido una recensión, vuelven a aparecer sobre todo en Ática y Eubea traidas por comerciantes fenicios en su búsqueda de los metales de estas regiones.

Pero será a partir del 760/50, del Geométrico Reciente, cuando asistamos a la definitiva expansión económica de Grecia, y a la recuperación de un verdadero comercio internacional.

A partir del 770 el Ática entra en una nueva fase de prosperidad económica. Hay un aumento de población y una expansión de la ciudad (Coldstream, 1977, 109). La actividad económica de Atenas se traduce ahora en la intensificación de la producción agrícola, especialmente del olivo, y metalúrgica, destinadas en gran parte a abastecer la demanda exterior. Llegan nuevas importaciones levantinas, intercambiadas por productos como el aceite, envasado en las famosas ánforas SOS que encontraremos por todo el Mediterráneo, otros productos agrícolas, y por la plata del Laurión. Sin embargo, sus producciones cerámicas, con los vasos del estilo del Dipylon a la cabeza, aunque alcanzan un nivel de calidad y de expresión simbólica monumental, no alcanzan el nivel de exportación del periodo anterior. Hacia el 730, durante el LGII, hay un cambio de orientación en Atenas, que prefiere explotar y colonizar su propio territorio antes que lanzarse decididamente a la aventura comercial (Coldstream, 1977, 109). Si a mediados del siglo vilı Atenas era aún una ciudad marítima con intereses comerciales, a partir del 730 los atenienses se concentran en la agricultura, y serán estos productos la base de la riqueza y prosperidad ateniense, que serán exportados y entrarán en las rutas comerciales internacionales a través no sólo de los comerciantes atenienses, sino también de otros intermediarios: fenicios, corintios y eubeos principalmente. 
El desarrollo económico de Eubea, sumamente evidente a partir de mediados del siglo $1 \mathrm{x}$, parece estar basado en dos factores de gran importancia: su ventajosa posicion geográfica, en las rutas que unen las zonas sur y norte del Egeo y, por tanto, con las rutas hacia las ricas zonas metaliferas del norte, $y$. especialmente, $y$ tal y como han propuesto investigaciones recientes (Bakhuizen, 1976), el desarrollo de una industria del hierro de alto nivel tecnológico basada en la explotación de las minas locales. Esta innovación tecnológica posiblemente permitió, entre otras ventajas, el incremento de los niveles de producción alimentaria con la mejora de las técnicas de explotación agricolas, y el consiguiente desarrollo demográfico, observable en el progresivo aumento de las zonas de habitación. La comercialización de excedentes y la posible exportación de productos de la industria local del hierro, bien en lingotes o en productos terminados (recordemos la fama, literaria, de las "espadas calcidias»: Bakhuizen, 1976, 43), favorecieron a partir del siglo ix el establecimiento de relaciones comerciales con los fenicios, a los que se debe la presencia en determinadas tumbas de Lefkandi del siglo $\mathrm{Ix}$ de ricas importaciones orientales: oro, marfil, fayenza (Coldstream, 1977, 63). Son todo estos elementos causas y signos de un progresiva prosperidad económica y de una cada vez mayor complejidad social que culminará en la segunda mitad del siglo viII.

Efectivamente, para Eubea, la segunda mitad del siglo viII representa el climax de su actividad comercial, que se extiende desde las costas levantinas hasta Italia Central. Es el momento de mayor auge, cuando la nueva ciudad de Eretria alcanza su mayor tamaño, cuando se construye el Hecatonpedón de Apolo Dafnéforo, cuando las tumbas de determinados representantes de grupos sociales dominantes, por ejemplo en la necrópolis de la Puerta Oeste de Eretria (Bérard, 1970), muestran una gran concentración de riqueza y una exhibición y consumo ostentoso de objetos de prestigio, cuando en esas mismas tumbas aparecen importaciones orientales - cerámica chipriota, escarabeos fenicios, calderos orientales-, cuando las producciones cerámicas eubeas, especialmente los escifos de pájaros, influidas por el estilo ático y el corintio (imitaciones de cotilas protocorintias), se difunden en gran cantidad y en una gran área, confirmando la vitalidad de su comercio y de su competitividad comercial, rasgo este último que se traduce en la imitación de las ánforas áticas de aceite SOS (Johnston y Jones, 1978). Los vasos exportados llegan a Delos, Naxos, Samos, Itaca, Zagora, Creta oriental y Macedonia. Las localidades chipriotas de Salamina, Kition, Amatonte, Kourion y Pafos fueron visitadas por comerciantes eubeos. Pero su principal puesto comercial en el Levante fue Al Mina, donde las cerámicas eubeas son muy frecuentes a partir del 740, y donde llegaron a inspirar imitaciones locales (Coldstream, 1977).

En cuanto a su expansión comercial hacia Occidente, los primeros signos de su interés por los ricos mercados centro italianos se datan en el periodo anterior, y se traducen en la llegada de vasos de estilo MGII: escifos de semicírculos colgantes y escifos de pájaros de una metopa. A partir de la creación del establecimiento de Pitecusa en 770 y de las primeras fundaciones coloniales a partir del 750 , la intensificación de la actividad comercial eubea en esta zona, en concurrencia con otros comerciantes griegos y orientales, dará un impulso extraordinario al comercio internacional mediterráneo, una de cuyas terminales será precisamente Pitecusa, pero de ello, hablaremos más adelante.

El comercio eubeo ofrece, en mi opinión, un matiz importante, diferenciado de otras poleis, que conviene destacar. Aparte de la importancia que pudo tener en él la industria del hierro, la comercialización de los excedentes agrícolas, o la exportación de su producción cerámica - que en ningún momento alcanza el nivel de la corintia-, creo que su papel podria entenderse también como de intermediarios, como agentes de articulación comercial de tres grandes áreas de producción e intercambio en esta época: el Levante, el Egeo, incluyendo sus regiones septentrionales donde los eubeos establecen redes comerciales secundarias, y el Mediterráneo central. Pero, al igual que en Corinto, esta actividad debió estar controlada por una minoría social, que, como hemos visto, a partir de este momento muestra sus mayores niveles de expresión de dominio.

En cuanto a Corinto, la tercera de las poleis en juego, se había convertido al comienzo de este periodo en el principal poder comercial de Grecia. Ya a comienzos del siglo VIII, durante el MGII, la expansión de la polis de Corinto coincide con un incremento de sus actividades exteriores. Los productos cerámicos corintios, especialmente los escifos de chevrones verticales, comienzan a documentarse en el Mediterráneo central, aunque quizás vehiculados por los eubeos en sus primeros movimientos exploratorios de las posibilidades de los ricos mercados centroitalianos (Coldstream, 1977, 167). Pero es a partir de mediados del siglo vilI cuando Corinto se lanza a la expansión comercial, como salida frente a un exiguo y escasamente productivo territorio, y aprovechando su ventajosa posición geográfica en el Istmo con acceso a dos mares. Es ahora cuando se constata un reforzamiento del sector agrario me- 
diante la adición de territorio, cuando se establece un puesto comercial en Itaca, cuando se funda el templo de Hera Limneia en Perachora (una importante zona para el control de la navegación en el Golfo de Corinto), cuando se fundan Siracusa (733 a.C.) y Corcira (709 a.C.), la primera llevada a cabo por un oikistés perteneciente a la familia oligárquica dominante. Es también ahora cuando se produce una intensificación en los niveles de producción de determinados productos, tanto naturales - especialmente el aceite - como manufacturados, y el desarrollo de una artesanía cada vez más especializada (Roebuck, 1984b), y todo ello consecuencia de una cada vez mayor demanda exterior estimulada por la expansión hacia el Occidente de los comerciantes corintios. La formación de grupos de poder que basan su dominio en el control de los medios de producción y de distribución, no sólo interior, sino cada vez con mayor fuerza, exterior, provocó un cambio en el poder político, hecho datado según Diodoro hacia el 747 , que ahora ya no está en manos de una monarquía hereditaria, sino de una cada vez más poderosa oligarquia mercantil, la de los Baquiadas.

El éxito comercial de Corinto se debe, entre otras causas, a la formación de un artesanado altamente especializado cuyos productos son altamente competitivos en los mercados internacionales. En primer lugar, por su volumen de producción, que podríamos considerar ya industrial, y comercialización, destacan las cerámicas finas, que se exportan ahora a los principales centros griegos continentales e insulares - donde influyen sobre el desarrollo de los estilos geométricos locales-, hacia el Levante y, sobre todo, al Mediterráneo central. Son estas producciones las primeras en recoger - en el llamado estilo protocorintio- los estímulos artísticos procedentes de Oriente, confiriendo al estilo corintio un dinamismo y vitalidad que Atenas, ya a fines del período, no supo mantener. Entre estas cerámicas destacan las cotilas hemiesféricas del tipo Aetos 666 y las copas de la clase de Thapsos, producidas desde el 750 y abundantemente representadas en Italia, $y$, más tarde, desde el 720 , las cotilas altas protocorintias y los aríbalos globulares, con una distribución igualmente amplia. Es una producción pensada básicamente para la exportación. Las copas del tipo Thapsos no han sido halladas en Corinto, sólo unas cuantas en Perachora; los vasos especializados como contenedores de perfumes, los aribalos, no aparecen tampoco en las zonas de habitación, necrópolis y santuarios locales. Ambos productos parecen ser respuestas especiales de los talleres corintios a las demandas abiertas por el mercado exterior (Roebuck, 1984b, 118-120).
En segundo lugar el trabajo del metal, que estimula el desarrollo de una escuela local de broncistas que realizan figuritas humanas y animales como elementos de adorno de los tripodes calderos de bronce, vasos de bronce, objetos de adorno personal, y armas, especialmente los famosos cascos. No menos importante fue el trabajo del marfil, especialmente de los sellos discoidales decorados con escenas figuradas, comparables en temas y calidad a los mejores vasos protocorintios. y con una amplia circulación, especialmente en los santuarios del Peloponeso e Itaca. Las manufacturas textiles y las terracotas arquitecturales también fueron elementos importantes en el comercio corintio, aunque fuera, al menos para las últimas, a nivel intra-regional y no internacional.

Junto a estas manufacturas debemos colocar otros productos corintios, cuya manufacturación, al menos en uno de los casos, indica también un alto grado de especialización artesanal: el aceite y el perfume. El aceite, envasado en las ya, desde este momento de finales del siglo viII, características ánforas del tipo A, comienza a exportarse por el Mediterráneo, y alcanzará regiones tan alejadas, como veremos más adelante, como la Península Ibérica, aunque en estos momentos no alcanza a competir todavía ventajosamente con el aceite ático. Pero fue el perfume el que se convirtió en una de las principales industrias corintias pensadas para la exportación, tal y como se deduce de la amplísima distribución de los típicos contenedores de perfume corintios, Los aribalos globulares, y su ausencia por estas fechas en los ajuares locales. Aunque quizás deberiamos matizar, como propone Coldstream $(1968,346$; Roebuck, 1984, 118), y decir que no sabemos realmente la procedencia de su contenido, es decir, dónde se elaboraba el perfume, y que posiblemente fuera importado a granel para ser reenvasado en Corinto, aunque si fuera así, ello indicaría una aún mayor especialización en la organización del comercio. Fuera donde fuese, el caso es que desde el 720 los comerciantes corintios están negociando activamente con este producto, en competición con los fenicios.

En resumen, el crecimiento económico de Corinto, derivó de su inmersión en el comercio internacional mediterráneo, pues ello permitió y provocó la intensificación de la explotación agraria, gracias a la colonización expansiva de su propio territorio, la comercialización de los excedentes de producción hacia el exterior y el surgimiento de una artesanía especializada, para responder al intercambio exterior. El grupo social dominante, las familias aristocráticas como la de los Baquíadas, que controla la posesión de las tierras y de los medios de produc- 
ción agricola y artesanal, y el comercio exterior. apoyado por iniciativas aristocráticas como la fundación de establecimientos y colonias en ultramar o el dominio de zonas como Perachora o Delfos, fue el que condujo el sinecismo de ('orinto, el que llevó a cabo la creación de la polis, como marco politico e institucional para el ejercicio de su dominio y mantenimiento de su posición de privilegio.

\section{EL LEVANTE. LA ACTIVIDAD COMERCIAL FENICIA}

Las causas de la expansión fenicia han sido ya expuestas por otros autores (Frankenstein, 1979; Aubet, 1987, con la bibliografia sobre el tema): las limitaciones topográficas de su territorio y su sobreexplotación y consiguiente caida en los rendimientos, el desequilibrio entre crecimiento demográfico y capacidad de los recursos locales, la necesidad de abstecimiento externo, el desarrollo de industrias de manufacturación especializadas para compensar las importaciones, $y$ la búsqueda de materias primas para atender a sus necesidades.

La historia de la expansión comercial de Tiro, intimamente ligada al desarrollo económico y politico del Próximo Oriente, comienza en el siglo $\mathrm{x}$, pero es en la segunda mitad del ix cuando los acontecimientos politicos, especialmente la presión del imperio asirio, imponen la reorganización de su estrategia comercial y su decidida "vocación occidental».

S. Frankenstein ya subrayó la estrecha interdependencia existente entre las ciudades fenicias y el imperio asirio, especialmente durante los siglos VIII y vil. Un factor esencial del imperialismo asirio fue el control sobre el comercio interegional: Asiria requería materias primas, especialmente metales, para sostener su desarrollada producción agrícola y su industria artesanal, y para sostener su máquina de guerra. El tributo impuesto sobre las ciudades fenicias fue más bien una imposición económica con vistas a reorientar su actividad económica y comercial hacia Asiria. Se convirtieron asi en los principales suministradores de materias primas al imperio asirio. Para atender las nuevas demandas dentro del sistema comercial regional, las ciudades fenicias se vieron forzadas a incrementar el nivel de producción de las manufacturas necesarias para los intercambios, a extender sus relaciones de intercambio y a extender su acción fuera de las redes comerciales tradicionales (Frankenstein, 1979). El cierre a los mercaderes de Tiro del acceso a los metales anatólicos fue un elemento más que marcó el viraje hacia Occidente de la politica económica y comer- cial de Tiro en busca de nuevas regiones metaliferas y nuevos mereados de abastecimiento de metales. La fundación de Kition a fines del siglo ix marca el primer punto en la expansión hacia Occidente.

Pero la presencia fenicia en aguas del Egeo fue anterior a la fundación de Kition y al establecimiento griego en Al Mina. Cuatro yacimientos egeos tienen una especial importancia para estos primeros signos de contactos con los comerciantes fenicios: la necrópolis de Kerameikos en Atenas, las necrópolis de Lefkandi, de Teke en Cnossos, y la de Seraglio en Cos. Creta y el Dodecaneso formaban parte de las usuales rutas comerciales del Mediterráneo oriental, pero Eubea y Ática deben considerarse desde el punto de vista fenicio como puntos terminales. La mayoria de los hallazgos datan de mediados del siglo Ix: una patera fenicia de bronce hallada en la tumba 42 del Kerameikos, objetos de de adorno de fayenza en la tumba de la Mujer rica del mismo cementerio. En Lefkandi son cientos los objetos de fayenza encontrados en las tumbas, entre ellos una figurita de Isis amamantando a Horus, y una figurita de Ptah-Osiris, sellos de esteatita de estilo egiptizante y escarabeos. También se encuentran los primeros objetos de oro: anillos y adornos para el pelo en forma espiral, importados de oriente (Coldstream, 1982b, 264).

Hay, por tanto, evidencias de comerciantes fenicios en el Egeo durante el siglo anterior a la fundación de Al Mina, en una época en que las importaciones griegas al Mediterráneo oriental eran bastante raras. Estos objetos de lujo orientales indican, más que visitas ocasionales, un intercambio comercial regular, aunque a pequeña escala. Sin embargo, evidencian un intercambio de alto nivel, pues el producto que los fenicios recibían a cambio no debia ser otro que la plata de las minas del Laurion, que comienzan a explotarse justo en este momento. Eubea no podia proporcionar directamente este metal precioso, pero si el hierro, y actuar como intermediario y abastecer la demanda fenicia a través de su red comercial establecida con Macedonia y Tesalia, es decir, con la plata de las regiones septentrionales del Egeo, y posiblemente esclavos.

Se ha sugerido que la presencia fenicia en el Egeo no consistió exclusivamente en el establecimiento de intercambios comerciales, sino también, lo que es más decisivo para el futuro desarrollo de una artesanía especializada griega - sin hablar de la adopción del alfabeto fenicio-, en el asentamiento de maestros joyeros orientales, sobre todo en Ática y Creta a partir del siglo Ix (Coldstream, 1982b, 266). A través de ellos se introduciría la técnica del granulado y diversas formas y motivos decorativos de 
carácter oriental, presentes en los objetos depositados en las tumbas más ricas de este periodo.

Otra forma de empresa fenicia en el Egeo, que implica tanto a comerciantes como a artesanos, se deduce de la presencia de importaciones y de imitaciones locales de diversas fabricas chipro-fenicias. especialmente en el Dodecaneso y Creta. Las formas son principalmente vasos de perfumes del tipo Black-on-Red. Las primeras importaciones de este tipo aparecen en Cos hacia el 850 . Pronto surgen las primeras imitaciones en el estilo $\mathrm{MG}$, pero también como contenedores de perfume. Parece que los fenicios habian establecido una pequeña factoria de perfumes en Cos donde envasaban el producto localmente con la ayuda de alfareros griegos. Lo mismo ocurrió en Knossos hacia el 800. Primero se creó la demanda a través de las importaciones, y luego se satisfizo con perfumes envasados localmente en una pequeña factoría llevada por fenicios residentes, con ayuda de alfareros griegos (Coldstream, 1982b, 268; Frankenstein, 1979, 275).

La asociación de fenicios y griegos en el comercio del perfume alcanza una nueva forma de expresión a mediados del siglo vIII. En Rodas se establece una factoria de perfumes que emplea alfareros orientales. Aquí los envases siguen muy de cerca los modelos orientales en fábrica, forma y decoración: además de las formas Black-on-Red, vemos copias bastante precisas de los oinocoes de boca de seta con engobe rojo, y jarros basados en el estilo chipriota White Painted IV de inspiración siria. Rodas, la isla más próxima a Fenicia, fue donde una comunidad fenicia de artesanos y comerciantes residentes pervivió más tiempo (Coldstream, 1982b, 269). De la importancia de Rodas en el comercio internacional, y de la conjunción de intereses comerciales griegos y fenicios, nos hablan también determinados tipos de objetos, como los sellos escaraboides del grupo del Tocador de Lira y los aríbalos ácromos, recientemente atribuidos a fábrica rodia, ampliamente distribuidos por el Mediterráneo oriental e Italia por agentes orientales (Martelli, 1991).

Esta temprana presencia fenicia en el Egeo, durante la época de formación de su poderio comercial, no tuvo el caracter de una empresa decidida y a gran escala. El comercio asiático le proporcionaba todavia los productos y rendimientos necesarios para el sostenimiento de su estructura y mecanismos. Cuando, a partir del siglo vili, la presión asiria imponga otra estrategia comercial, y especialmente la necesidad imperiosa de suministrar plata en grandes cantidades, la actividad fenicia no se dirigirá al Egeo, sino hacia el Mediterráneo central y, sobre todo, al extremo Occidente, donde los recursos eran suficientemente importantes, y los poderes locales suficientemente débiles, o, mejor dicho, las economias perifëricas, como para producir pingües beneficios y sustentar la gran inversión que supone el comercio a larga distancia.

\section{GRIEGOS Y FENICIOS EN EL MEDITERRÄNEO CENTRAL}

Durante la primera mitad del siglo vin, y antes de la fundación de las primeras colonias, los comerciantes eubeos habian penetrado en el Mar Tirreno y comerciaban con los habitantes de Etruria y Campania. La atracción fundamental de estas regiones era la abundancia de metales, especialmente hierro. Encontramos a lo largo de las costas tirrénicas de la península itálica importaciones eubeas del MGII: escifos de chevrones o de pájaro en Pontecagnano, Capua, Veyes y Tarquinia, y una cratera en Pescia Romana (Coldstream, 1977. 223). En las costas meridionales, cuyo atractivo se basaba en los productos agricolas y ganaderos, hay indicios de la temprana presencia eubea en Incoronata, donde encontramos un escifo de chevrones MGII y una protocotila corintia, así como en Otranto, donde también encontramos numerosas importaciones griegas del MGII. En Sicilia las importaciones de Villasmundo, especialmente un escifo de semicírculos colgantes $y$ otro de chevrones, también nos informan de los contactos de las poblaciones locales con los comerciantes eubeos en la primera mitad del siglo VIII (Coldstream, 1977, 233).

Hacia 770 los eubeos establecen Pitecusa, el primer puesto comercial permanente griego, uno de los grandes centros de intercambio internacional del Mediterráneo central durante el siglo viII. Su situación geográfica era enormemente favorable, a las puertas de las rutas que conducen hacia las ricas fuentes del metal de Elba en el norte de Etruria, y de las rutas de abastecimiento y circulación del mineral y que conectan con el inmenso y rico mundo de la Europa central, en la costa de una fértil región - Campania - donde también los productos agricolas podrian constituir un elemento de intercambio, aunque en estos primeros momentos no a tan gran escala como el metal, y a las puertas de un amplio y rico mundo de poblaciones indigenas que podian constituir un foco de demanda y consumo de productos griegos de extraordinarias posibilidades. $\mathrm{Y}$ también, en un punto neurálgico de comunicaciones, donde confluirán diversas rutas del comercio internacional mediterráneo.

El carácter mercantil de este primer asentamien- 
to se deduce de la existencia, desde el primer momento de su fundacion, de un barrio dedicado a la fundición y trabajo artesanal del metal, bronce, plomo y hierro, metales procedentes de la región etrusca, particularmente de Elba, y de los hallazgos de su necrópolis. Son especialmente estas importaciones las que nos proporcionan una mejor idea del caracter internacional de este centro: además de las lógicas producciones eubeas, encontramos cerámica corintia. Ambos tipos fueron imitados localmente y encontramos producciones pitecusanas de cotilas de chevrones y oinocoes LG del tipo corintio, y producciones pitecusanas de escifos y anforas del tipo eubeo. Junto a ellas se documentan importaciones rodias, especialmente vasos de perfumes y cotilas de pájaros, ánforas áticas SOS, ánforas corintias A y cerámica argiva monócroma (Ridgway, 1982). Encontramos también fïbulas greco-orientales del tipo "anatólico» y una impresión de un sello en un ánfora que corresponde a un sello del tipo plano y cuadrado hecho en Argólida y las Cícladas. También son frecuentes las importaciones orientales: jarros de boca de seta con engobe rojo fenicios, aribalos nord-sirios, 87 sellos del grupo del Tocador de Lira, unos 100 escarabeos, los más antiguos imitaciones fenicias, pero los de fines del VIII originales egipcios (Büchner, 1982). Coldstream sugiere que posiblemente fueron llevadas hasta allí por los propios comerciantes eubeos, que podrian haberlas adquirido en sus puestos comerciales levantinos, y que a través de ellos habrían llegado algunas de estas importaciones a Etruria (Coldstream, 1977, 226), mientras los Sherratt defienden unos contactos fenicios con Etruria indirectos, a través de Cerdeña (Sherratt, 1993, 365). Por otra parte, Martelli defiende una distribución por agentes orientales de los objetos rodios, especialmente los aribalos ácromos, las «vogelperlen» en pasta vitrea y los sellos escaraboides del grupo del Tocador de Lira, cuya distribución en Grecia, muy limitada, y en el Mediterráneo Oriental apuntan a los comerciantes semitas y a su actividad en el puerto de Rodas, en probable conexión con la compañia oriental allí instalada desde mediados del IX (Martelli, 1991, 1050). Además, la fuerte presencia de cerámicas de engobe rojo en la misma Pitecusa, y además cerámicas de "sabor» fenicio-occidental, especialmente los platos con tondo en reserva similares a los de Huelva (Fernández Jurado, 1987, 356), nos hablarian de un interés y una presencia fenicia activa en esta región, extendiéndose hasta Etruria, y actuando desde sus bases en el Mediterráneo central: Sicilia, Cartago o Cerdeña. La presencia de materiales fenicios en Populonia (Martelli, 1981, 404), en la Etruria septentrional, en las mismas fuentes del metal, es un argumento más en favor de esta idea.

La misma variedad de importaciones se encuentra en (umas, la colonia eubea fundada hacia el 750 en tierra firme, frente a Pitecusa. Junto a la cerámica eubea encontramos cerámica corintia, imitaciones pitecusanas y algunos aribalos rodios. Entre los objetos de metal, destacan las fibulas de plata de tipo etrusco y otros objetos de adorno personal. Son numerosas también las importaciones orientales: escarabeos, sellos Nord-sirios y un caldero de bronce con prótomos de toro de tipo urartiano o nordsirio, muy similar al hallado en la tumba principesca de la Puerta Oeste de Eretria, donde posiblemente llegaria a través de estos centros mercantiles (Coldstream, 1977, 231).

Es precisamente a partir de la segunda mitad del siglo vIII, cuando se introducen también en los ajuares etruscos importaciones griegas (Coldstream, 1977. 232). El mercado etrusco integra estos productos griegos en un esquema de valor de alto nivel, como productos de lujo, pues tanto las cerámicas finas como el vino y el aceite griegos aparecen precisamente en las tumbas principescas, como la Regolini Galassi, Capanna y Monte Albatone 4 de Cerveteri (Rizzo, 1990, 27).

Hagamos ahora un rápido repaso de la actividad fenicia en el Mediterráneo central. Aunque las actividades comerciales levantinas en esta región, en conexión con el tráfico de metales con base en Cerdeña, se pueden rastrear desde el siglo $\mathrm{x}$ (Niemeyer, 1984; Strom, 1991), nos centraremos en el momento del establecimiento de relaciones y centros permanentes, es decir, el siglo vill.

Según las fuentes escritas los tirios fundan Cartago en el 814 a.C., pero los hallazgos más antiguos de la necrópolis de Juno datan de hacia el 730 . Sin embargo, el hallazgo en el tofet de Salammbo, bajo los primeros niveles de urnas del tofet, de una pequeña cámara con ofrendas entre las que se encontraban importaciones griegas de mediados del VIII, acercó la cronologia a las fechas históricas de la fundación. Las importaciones halladas consistian en cerámica eubea y corintia (cotilas EPC), procedente de Pitecusa. Aunque Coldstream hablaba de un grupo homogéneo de vasos datados entre 740-710 (Coldstream, 1977, 240), Bisi defiende la hetereogeneidad de estas importaciones del depósito de fundación del tophet, donde ve dos componentes, uno eubeo anterior, y otro corintio más tardío, en un arco cronológico que se extiende desde el 770 al 680 (Bisi, 1983, 712). Recientemente, nuevas excavaciones han repetido el hallazgo de importaciones geométricas, incluso algo más antiguas: una cotila 
del tipo Aetos 666 y un escifo corintio de chevrones del MGII, además de los escifos de pájaros eubeos. ya de la segunda mitad del vill (Vegas, 1989).

La presencia de estas importaciones del MGill ha hecho pensar a Bisi que hay una fase en la que los fenicios debieron frecuentar las rutas hacia el mar Tirreno paralelemente al tráfico precolonial griego hacia las costas de Etruria, Lacio y Campania, en un momento anterior a la fundación de Pitecusa (Bisi, 1982). La presencia en Tarquinia de objetos de fayenza, un escarabeo en esteatita y una jarra con boca discoidal bicroma nord-siria, en contextos de fines del Ix y comienzos del VIII, hablan en favor de esta idea (Martelli, 1991, 1056).

A fines del siglo viI los fenicios fundan Motya en la costa occidental de Sicilia. Aqui también se encuentran importaciones e imitaciones griegas fechadas entre 720-710: aribalos EPC, cotilas, y escifos del tipo Thapsos, e imitaciones fenicias de escifos subgeométricos.

En Sulcis (Cerdeña), donde los fenicios se habian establecido desde mediados del VIII, de nuevo encontramos importaciones geométricas (Bernardini, 1988): fragmentos de cotilas del tipo Aetos 666 de fabricación pitecusana, fragmentos de oinocoes, un escifo de chevrones - en un contexto posterior al 750 - asi como producciones locales de clara inspiración eubea. Junto a ellos, un numeroso grupo de copas escifoides de fabricación fenicia, que asume directamente del Geométrico Final griego su inspiración decorativa y formal, un grupo también representado en las colonias fenicias de Occidente, donde son especialmente abundantes (Rouillard, 1990; Briese y Docter, 1992). Los hallazgos de Sulcis prueban la estrecha relación existente entre este establecimiento fenicio y Pitecusa, demostrando, una vez más, la conjunción de intereses comerciales entre eubeos y fenicios en esta ruta que conduce desde el Levante al Mediterráneo occidental (Bernardini, 1988, 1991).

Todos estos datos sugieren que debió existir un interés comercial fenicio en las regiones centro-italianas, a las que acudian desde sus enclaves estratégicos situados en puntos desde donde controlaban las principales rutas comerciales que unian el Levante y el Egeo con el Mediterráneo central y occidental. En realidad podriamos hablar de una concurrencia de intereses entre fenicios y griegos por acceder a las ricas posibilidades que les ofrecia el mercado etrusco en cuanto a la adquisición de materias primas y a la salida de sus propias manufacturas. La ruta hacia esta zona está marcada por hallazgos - datados en el primer cuarto del vill por Niemeyer, y en los años centrales del vin por Mar- telli: Niemeyer, 1984; Martelli, 1991- de FrancaviIla Maritima y Pitecusa. El acceso al mercado etrusco por parte de los fenicios ya en la segunda mitad del siglo vili se constata en la presencia de escarabeos del grupo del Tocador de Lira rodios - si admitimos con Martelli su vehiculación por agentes orientales, y no por los mismos griegos de Pitecusa - y una patera fenicia de bronce del tercer cuarto del vill en Vetulonia, o en los escarabeos de fayenza y el riton de bronce asirio de fines del siglo en Veyes, o en la situla de fayenza con el escarabeo del faraón Bocchoris encontrada Tarquinia, gemela de otra de Motya, o el cuenco tripode de Castel de Decima (Martelli, 1991), o el material fenicio de Populonia (Martelli, 1981; Rizzo, 1991, 1170). Lo dificil es saber si ese acceso se realizó directamente o a través de Pitecusa, donde cada vez son más numerosos los elementos que pueden constatar una presencia comercial fenicia, incluso anforas con inscripciones que apuntan la presencia de comerciantes fenicios (Büchner, 1982, 292, figuras 15-16), presencia que los materiales griegos y fenicios de España ayudan a confirmar (Cabrera, 1985, 1986).

Estos centros fenicios asentados en el Mediterráneo central, en el triángulo formado por Sicilia occidental, Cerdeña y el Golfo de Túnez, con el centro neurálgico en Cartago, proporcionarán acceso también a las redes comerciales indigenas que alcanzaban el área atlántica a través del Sur de España, Sur de Francia y Baleares (Ruiz Gálvez, 1986).

Pitecusa fue una de las puertas, aunque no la única, a través de la cual Etruria se integró, y con ella sus redes comerciales con Europa central, en el comercio internacional mediterráneo. El establecimiento de relaciones de intercambio con el Oriente y el Egeo pondrá en marcha una serie de mecanismos de interacción que darán como resultado un cambio en las estructuras socio-económicas etruscas. En palabras de D'Agostino, «El contacto con una sociedad más avanzada, de las que estas cerámicas griegas son el signo tangible, no fue ajeno al cambio operado en el terreno socio-económico del mundo proto-etrusco. Pero si este mundo no hubiera contenido en si mismo la premisa de una fuerte cohesión política y una relativa dinámica estructural, el impacto podia haber tenido un efecto muy diferente» (D'Agostino, 1985, 46)

Estas transformaciones culturales y sociales se podrían explicar, con la matización final de D'Agostino, en términos de respuesta de una periferia al impacto de un área central, de forma que el desarrollo político de Etruria se entendería como una consecuencia natural de su subordinación económica al área central egea y oriental. Sin embargo, Stod- 
dart (1989) recientemente ha criticado esta vision "simplista" de Etruria como periferia, visión que considera las relaciones entre etruscos y orientales asimétricas en términos de desarrollo económico. En realidad no hay evidencias de extraccion de recursos de un área en beneficio exclusivo de otra. Hay una estructuración politica y económica territorial en el periodo villanoviano, con areas especializadas en diferentes tipos de producción, que marcó la ruptura en la uniformidad cultural y politica de Italia central. Sin embargo esta especialización no denota una dependencia económica de un área con respecto a otra en el sentido estructural marcado por la relación centro-periferia. El segundo desarrollo político, operado en el siglo viII, es un proceso gradual, continuación de lo iniciado en el periodo anterior. Los centros de poder etruscos simplemente explotaron la oportunidad presentada por la presencia de comerciantes mediterráneos, y de los productos que introducen, para adquirir un nuevo medio de expresión de estatus y consolidar el cambio politico. La importancia de la extracción de metales es reducida y no es causa del desarrollo socio-político (Stoddart, 1989).

\section{LA PENINSULA IBÉRICA}

En palabras de M. E. Aubet, «el comercio de la plata fue el objetivo principal de la expansión fenicia al extremo Occidente. $\mathrm{Y}$ obtención y producción de plata equivale a hablar de Gadir y de su hinterland inmediato, Tartessos»" (Aubet, 1987, 228). El origen de las primeras expediciones fenicias a occidente, por tanto, aparece relacionado con la riqueza argentifera de la peninsula ibérica $y$, especialmente, de Tartessos. Tiro iba a ser la primera en explotar y rentabilizar todo ese potencial económico.

La instalación de las primeras factorías o establecimientos fenicios en el sur de la península ibérica data de comienzos del siglo viI. El primer establecimiento fue Cádiz, fundado a comienzos del siglo, según testimonian las excavaciones recientes en el Castillo de Doña. Blanca, una fundación fenicia contemporánea de Cádiz. En torno a la bahía, una zona intensamente habitada por población indigena, se desplegó un entramado político y económico distribuido en tres puntos: el santuario de Melqart en la isla actual de Sancti Petri, con un importante significado religioso y económico, la ciudad de Gadir, como metrópolis y centro político administrativo, y el Castillo de Doña Blanca, próximo al poblamiento indigena de la campiña, como avanzadilla y punto de comercio (Ruiz Mata, 1993, 45).
A partir del segundo cuarto del vill surgen diversos asentamientos fenicios en la costa meridional mediterránea, desde Málaga a Almeria: Morro de Mezquitilla, Chorreras, Toscanos, Guadalhorce, Almunecar y Adra. El establecimiento de estas colonias fenicias se explica en función de Gadir, como centros de apoyo al control del acceso a los metales del área tartésica, y como centros agricolas. Pero su principal valor económico y politico, además de dar solución a la crisis demográfica de la metrópolis y a su fundamental carencia de recursos agricolas, residió en su papel como centros desde donde se controlaba, y monopolizaba, el acceso a los metales meridionales. Sin embargo, sin un aprovechamiento intensivo de los recursos del territorio inmediato no se justificaria una permanencia prolongada de una población oriental tan densa. Son pequeños centros autárquicos dotados de territorio y de recursos propios, que comercian con las poblaciones del interior, aunque a pequeña escala, y que basan su riqueza en la explotación agricola, ganadera y marítima, y en su vinculación al gran centro comercial que fue Gadir (Aubet, 1987).

Es en este marco donde se inscriben las importaciones griegas geométricas, de las que quisiera hablar con cierto detenimiento, pues son signos evidentes de la complejidad de rutas y de la internacionalización del comercio de este momento.

En primer lugar las halladas en el área tartésica, en Huelva. De alli procede el fragmento más antiguo hallado en la Peninsula, aunque desgraciadamente fuera de contexto: una pixida ática del MGII. Si hace algunos años yo misma proponía un retraso en su llegada a Huelva, basándome en su presencia en contextos de la segunda mitad del vIII en yacimientos del Próximo Oriente, y en la posible larga «vida» y circulación de un objeto de prestigio (Cabrera, 1986, 1988-89), hoy en día creo que podemos aceptar su llegada a Huelva en la fecha de su fabricación, es decir, en una fecha cercana al 770760. Tenemos hoy más documentos que permiten sustentar esta hipótesis: los hallazgos de cerámicas MGII en yacimientos fenicios centromediterráneos. Realmente, éste es el periodo de máxima expansión de los vasos áticos, y es el momento en que se produce el acercamiento de los fenicios peninsulares al mundo tartésico, a través de, como dijimos antes, de un sistema de intercambio basado en la reciprocidad, en el intercambio de dones, de objetos de lujo - la píxida ática-, y de prestación de servicios altamente especializados: la construcción del muro de sillares del Cabezo de San Pedro.

Pero en Huelva se han producido también otros hallazgos griegos de época geométrica: dos escifos 
eubeos de la segunda mitad del vill y una cotila del $E P C$, del último cuarto del vill. Debemos señalar que, hasta la fecha estas son las únicas importaciones griegas halladas en la ciudad de Huelva y en las proximidades, datables con seguridad en época geométrica.

Además de estas importaciones halladas en el área tartésica, conocemos ya un número relativamente importante de vasos griegos en las colonias fenicias. En primer lugar, y como datos inéditos, presentaré los hallazgos realizados en el Castillo de Dria. Blanca. Se trata de un fragmento de un escifo eubeo, que por su tamaño no podemos encuadrar en ningún tipo concreto, pero que sin duda es LG. En segundo lugar un ánfora corintia del tipo $\mathrm{A}$, hallada en niveles perfectamente fechados en la segunda mitad del viII, y un ánfora ática SOS del mismo momento. Hay otros dos fragmentos geométricos recientemente recogidos y que aún estoy estudiando, por lo que no puedo adelantar su fábrica ni su tipo.

En las recientes excavaciones realizadas en el Cerro del Villar, en Guadalhorce, se ha encontrado un ejemplar casi completo de ánfora SOS, pero no del tipo ático, sino eubeo, de fines del VIII (Cabrera, en prensa), del mismo tipo que algunas encontradas en Italia.

Los únicos ejemplares protocorintios que se han hallado completos son las dos cotilas de la tumba 19 de la necrópolis Laurita en Almuñecar, una de ellas del EPC, de fines del VIII, y otra del primer cuarto del vil, considerada una cotila de imitación posiblemente realizada en Pitecusa (Cabrera, 1988-89).

En Toscanos se han hallado fragmentos de cotilas protocorintias, pero aquellos con la zona inferior negra han aparecido en los estratos IVA y IVB, mientras que aquellos con decoración orientalizante con campo reservado y radios sobre el pie han aparecido en los estratos IVC y V, más tardios. Los primeros fragmentos podrian ser del EPC, del último cuarto del siglo vill; los segundos del MPC, de la primera mitad del siglo vil a.C.

Un dato interesante proporcionado por los hallazgos de Toscanos es que entre ellos también se encuentran fragmentos que pertenecen a cotilas de imitación realizadas en Pitecusa, completando así el dato proporcionado por la necrópolis Laurita sobre las relaciones, cada vez más evidentes, entre la colonia eubea y las colonias fenicias occidentales.

Junto a las cotilas de Almuñecar y Toscanos hay que situar otro fragmento hallado en el Cerro del Peñón, perteneciente a una cotila protocorintia, pero de dificil fechación, aunque probablemente de la primera mitad del itl, pues no se han encontrado cerámicas fenicias más antiguas.

Junto a las importaciones protocorintias se sitúan las importaciones de ánforas áticas SOS. En Toscanos aparecieron en los estratos IVA-B y IVB. Los fragmentos publicados corresponden a la zona del cuello, con la tipica decoración SOS. y con el caracteristico listel en relieve bajo el borde, a la zona del hombro con bandas reservadas, y a la zona del pie. Niemeyer fecha el más antiguo a fines del viII, y el resto a comienzos del vil. Pero, teniendo en cuenta que, como proponiamos más arriba, Toscanos IV se puede subir al siglo viII, debemos considerar una fecha más antigua para todos ellos.

Este conjunto de importaciones griegas nos permite esbozar ahora un panorama más preciso de los circuitos comerciales en los que se integran. Si analizamos las importaciones protocorintias y las ánforas SOS áticas, vemos que hay una especial concentración de estos productos en el Mediterráneo central, de nuevo en Italia. De hecho, las cotilas protocorintias tuvieron una amplia circulación en Italia continental y en Sicilia, pero las ánforas SOS se concentran en gran número en Pitecusa, donde además fueron imitadas localmente. Shefton ya apuntaba que fue en este punto donde los fenicios consiguieron el aceite ático que luego llevaron a las factorias del área del Estrecho, asi como las cotilas protocorintias y las cotilas de imitación pitecusanas halladas en Almuñecar y Toscanos (Shefton, 1982). En efecto, creemos que los datos que hoy poseemos confirman esta idea, pues los escifos eubeos y el ánfora eubea de Guadalhorce apuntan hacia esa misma región de Italia central.

¿Cuál es el valor de las importaciones griegas en el contexto social peninsular? En general, podemos decir que el valor de un producto forma el criterio principal para su significación simbólica. Los productos de riqueza son las afirmaciones principales del estatus social. Pero ¿cuál es el criterio para calificar un objeto como producto de lujo? Depende del contexto, pero en general podemos invocar varios criterios. Según Appadurai (1986), los productos de lujo son bienes cuyo principal uso es retórico y social, son signos encarnados. La necesidad a la que responden es fundamentalmente política y tienen los siguientes atributos: restricción a las élites, complejidad de su adquisición - en función o no de una «escasez» real-, virtuosismo semiótico, es decir, capacidad de contener mensajes socialmente complejos, conocimiento especializado como prerequisito básico para su consumo «apropiado», y alto grado de vinculación de su consumo al cuerpo, persona o personalidad. Podriamos añadir también, con $\mathrm{Me}$ - 
las (1991), el alto costo de su producción, los costes del transporte y su caracter exótico.

Las importaciones griegas en Tartessos se integran en el esquema economico y social fenicio, como productos de lujo que forman parte de las relaciones de reciprocidad basadas en un sistema de intercambio de regalos con las élites tartésicas a cambio de la plata.

El resto de las importaciones aparecen en contextos de hábitat - Torre de Doña Blanca-, en almacenes comerciales - en Toscanos y posiblemente Guadalhorce - 0 indefinidos - Cerro del PeñonPero, precisamente, la existencia de estos grandes almacenes, o centros comerciales como la Torre de Dña. Blanca, denotan la existencia de una organización administrativa, pública y mercantil perfectamente coordinada y centralizada, asi como la presencia de una «burguesia» mercantil especializada y altamente cualificada (Aubet, 1987). Las importaciones griegas deben verse como productos consumibles por esta "burguesía», como productos de prestigio que, por su exotismo y calidad intrinseca, se apartan de la tónica general de otras producciones cerámicas. La forma y el contenido de las necrópolis parece apuntar en el mismo sentido.

En la necrópolis Laurita, de las 17 tumbas publicadas (Pellicer, 1962), todas tienen como urna cineraria un vaso de alabastro o de mármol de fabricación egipcia, piezas excepcionales, que en origen contenian vino de calidad, procedentes del saqueo de tumbas reales egipcias o quizás procedentes de regalos ofrecidos por los faraones al rey de Tiro. Hay, por tanto, una cierta uniformidad e igualdad en las tumbas en base al elemento principal del ajuar funerario. Las diferencias entre ellas son más sutiles. Pero, precisamente, la tumba que contenía las dos cotilas protocorintias es la única que tenía un ajuar más completo: el conjunto típico «jarro de boca de seta-jarro piriforme», y un huevo de avestruz, además de la cerámica griega, y es la única que contenia un fragmento de hierro. Y si reconocemos el valor simbólico y de prestigio del oro o la plata, debemos reconocer también el del hierro en este momento. Aunque hay tumbas con pequeños elementos de oro o plata, son aquellas que no tienen conjunto típico ni huevos de avestruz. El conjunto típico aparece en otras tres tumbas, de las que sólo una contenia metal: un pequeño aro de bronce. Podemos, por tanto, permitirnos el establecer un valor elevado para los vasos griegos, pues aparecen en una de las tumbas más significativas desde el punto de vista simbólico y de prestigio.

En Trayamar no aparecen las importaciones griegas, pero ello quizás se deba, entre otras posibles razones, a que aqui la ostentación y manifestación de dominio no se expresa tanto a través del ajuar. como a través de la monumentalización del espacio funerario, de una arquitectura especializada al servicio de una familia que pertenece a la oligarquía mercantil. De todas formas, sólo nuevos hallazgos en contextos bien definidos, nos ayudarán a precisar estas cuestiones, que hoy por hoy sólo podemos apuntar y sugerir a nivel de hipótesis.

¿Qué repercusiones tuvo el comercio y colonización fenicia para el desarrollo de la sociedad indigena tartésica?

Tartessos fue el lugar hacia el que se dirigió la búsqueda de metales, y donde los fenicios, en conjunción con los poderes locales, establecieron todo un sistema comercial basado en la explotación de las minas, la elaboración del mineral y su canalización hacia los centros de comercialización. Los fenicios provocaron un cambio en cierto sector de la actividad económica de esta región: frente a una metalurgia basada exclusivamente en el cobre durante el Bronce Pleno y Reciente, será ahora, a partir del siglo vIII, cuando por primera vez se documente una actividad metalúrgica orientada a la explotación de las minas de plomo argentifero de la zona de Sierra Morena para el beneficio de la plata (Ruiz Mata, 1989, 235). Pero no sólo se produce un cambio de actividad extractiva y productiva, sino también un salto cualitativo y cuantitativo. La intensificación y el cambio en la producción sólo se pudo producir, con resultados económicamente ventajosos, gracias a la introducción por parte de los fenicios de novedades tecnológicas, y entre ellas la de la copelación, que permitia el beneficio de la plata y su rápida separación del plomo (Ruiz Mata, 1987, 235).

El control de estos medios de producción estaba en manos de un sector minoritario y dominante de la sociedad tartésica, cuya fisionomía no es facil de conocer con precisión. La ausencia de enterramientos del Bronce Final anteriores al establecimiento de relaciones con el mundo fenicio impide conocer determinados aspectos de la estructura social y, en especial, la posible existencia de un acceso diferenciado a la riqueza. Quizás, como se ha propuesto, las estelas del SO. reflejan la existencia de élites sociales, quizás caudillos guerreros, en un momento avanzado del Bronce Final (Barceló, 1989, 205), e inducen a hablar de una sociedad segmentada. Sin embargo, la excavación de un túmulo funerario en la necrópolis de Las Cumbres, junto al Castillo de Doña. Blanca, con enterramientos indigenas anteriores a la presencia fenicia, y otros donde se integran ajuares fenicios, que estuvo en uso a lo largo 
del siglo vIII, indica la existencia "de una sociedad incipientemente estratificada, que participa colectivamente en el hecho critico de la muerte de uno de sus miembros. Asi sugiere el mismo componente de los ajuares, con escasas diferencias, y desde luego tampoco se advierten grandes diferencias en la propia estructura de los enterramientos. No obstante, en los momentos finales se perciben atisbos de resaltar diferencias sociales" (Ruiz Mata y Pérez. 1989, 292). Será, por tanto, a partir del establecimiento de relaciones con los fenicios cuando se constate una clara jerarquización social y un acceso claramente diferenciado a la riqueza, proceso que se agudiza a partir del siglo vil, como denotan la estructura monumental y ajuares ostentosos de las tumbas "principescas» tartésicas (Aubet, 1984).

Es la actividad comercial, y la inmersion de las élites tartésicas en el comercio internacional mediterráneo la que origina la aceleración del proceso de cambio y una rápida transformación de los sistemas económicos y sociales, y una nueva estructuración social basada en un cada vez mayor desigualdad entre sus miembros. Asímismo, consolidó una estructuración y jerarquización del territorio en función de la actividad metalúrgica y en función de su comercialización a través de Huelva y de Cádiz, principal receptor de la plata tartésica, y detentador del monopolio de su comercialización en el Mediterráneo.

Los comerciantes fenicios impusieron sobre las élites tartésicas, que controlaban los medios de producción, la demanda de consumo de nuevos productos, altamente significativos, que, al introducirse en una nueva "esfera de intercambio» adquieren una nueva significación como bienes de prestigio. La necesidad de consumir productos de lujo para la reproducción del sistema social, motivó la intensificación de la producción, muy especialmente metalúrgica, aunque también agropecuaria, y la extracción de excedentes, que proporcionaron bienes para el intercambio. La legitimación y consolidación de las estructuras de dominio necesitaron de la importación de productos como textiles, perfume, orfebrería, marfiles, vasos de alabastro, huevos de avestruz, bronces, vajilla cerámica fenicia $-\mathrm{y}$ griega, aunque en menor medida - y vino y aceite, que sólo se podian adquirir a cambio de productos de la metalurgia, especialmente la plata.

Los productos intercambiados por la plata tartésica denotan la existencia de un sistema de comercio típicamente colonial: la producción de auténticos artículos para la "exportación», como son los envases de perfumes, $y$ una serie de objetos de lujo y prestigio - joyas, marfiles, bronces -, o las mismas importaciones griegas, destinados a la élite tartési- ca. La concentración. especialmente evidente en el siglo posterior, de productos de lujo o dones de prestigio en zonas estratégicas como Huelva, Carmona, Carambolo. Setefilla, Aliseda o Cástulo, y formando parte de ajuares funerarios principescos, demuestra el interés de Gadir hacia las poblaciones que controlaban las principales vias de comunicación y accesos hacia los recursos mineros y agropecuarios del interior. "Una constante del comercio en la Baja Andalucia fue el sistema de reciprocidad, que aparece circunscrito a los sectores privilegiados de la sociedad tartésica. Y el intercambio de metales por vino, aceite y perfumes es indice de un poder desigual y de una situación típicamente colonialista. Una situación en la que el intercambio se lleva a cambio entre sociedades desiguales, sólo podia traer dos consecuencias también tipicamente coloniales: un cambio social en el seno de la sociedad indigena, desde el momento en que determinados sectores de la población se incorporan al circuito comercial fenicio y se benefician de la situación, y un agotamiento de los recursos del territorio a largo plazo.» (Aubet, 1987, 253).

En definitiva, los fenicios establecieron en el Occidente una esfera comercial que se convirtió en una nueva área periférica, donde iniciaron la explotación de ciertos recursos e incrementaron la explotación de otros. A través de la élite local como intermediaria, el excedente de los recursos domésticos fue producido para el consumo fenicio. Las relaciones de los fenicios con la población indigena produjo cambios en el seno de estas sociedades en términos de dependencia económica y politica dentro de un sistema comercial tanto regional como internacional. Los fenicios, en palabras de S.Frankenstein, impulsaron la incorporación inicial del Sur de la Peninsula en la red comercial mediterránea como una periferia dentro del sistema regional asiático occidental (Frankenstein, 1979, 290).

Quisiera finalizar haciendo un balance de todo lo expuesto hasta ahora, y resumir el papel de las importaciones griegas en el marco del comercio internacional mediterráneo del siglo vIII. Las importaciones españolas son un elemento más, junto con las del área italiana o levantina, y cada una en su contexto, que nos ha permitido acercarnos a ese complejo mundo del comercio y de los intercambios. A través de ellas, y repito que como uno de los muchos elementos que forman parte de este proceso, extraemos las siguientes informaciones:

1. La recuperación, por primera vez desde el colapso del sistema comercial de fines de la Edad del Bronce, de un verdadero comercio internacional a gran escala. 
2. La expansión y crecimiento de los centros, primero en el Levante. más tarde en el Egeo, en un movimiento progresiso desde Oriente a Occidente. y que implicará a un número cada vez más amplio de comunidades y de àreas geográficas.

3. La existencia en este comercio internacional mediterráneo del siglo vil de un complejo entramado, con diversas redes comerciales establecidas entre el Levante, Girecia. Mediterráneo central y Mediterráneo occidental, así como redes comerciales secundarias, que amplian considerablemente el radio de acción.

4. La existencia de un mercado internacional de metales, con grandes centros de intercambio en diferentes zonas, que a su vez establecen redes comerciales secundarias dependientes, siendo el metal el articulo que hizo posible la articulación entre los mercados locales y los mercados interegionales.

5. El protagonismo decidido en este comercio internacional de fenicios y griegos, particularmente eubeos y, quizás desde un poco más tarde, corintios, en una simbiosis comercial donde, de momento, no hay rutas ni areas restringidas, sino concurrencia de intereses y posiblemente colaboración, llegándose a hablar de "aventura conjunta» (Sherratt, 1993). Sin embargo se anuncia ya la competición comercial y la necesidad de definición territorial, y de acceso exclusivo a determinadas materias primas, a través de la fundación de colonias, proceso que en el siglo siguiente dará lugar a la definición de rutas exclusivas (Sherratt, 1993).

6. La participación en este sistema de intercambio de productos manufacturados que, al entrar en redes comerciales diferentes, adquieren un valor diferente - por ejemplo, las cerámicas griegas en las redes comerciales secundarias fenicias-.

7. La «internacionalización» de determinados contenidos semánticos - sociales, religiosos, estéticos, que acompañan a los productos intercambiados, contenidos que circulan de un extremo a otro del Mediterráneo, y que penetran en el tejido cultural en diferentes grados y a diferentes niveles -en las periferias no penetrarán más allá, como los mismos productos, del nivel de la élite que los demanda-, koiné que será más evidente y generalizada a partir del siglo vil.

8. La aceleración, como consecuencia de este comercio internacional, de un proceso de cambios sociales y económicos que afectan tanto a los grandes centros - Levante, Grecia, y en una ampliación del centro, las colonias fenicias y griegas - como a las periferias - especialmente, por lo que nos atañe, la sociedad indigena peninsular-:

- Consolidación de clases dominantes que ba- san su poder en el control de los medios de producción y/o distribución interior y exterior de los excedentes, $y$ legitiman su predominio en el prestigio que les confiere el intercambio y acumulación de bienes de lujo.

- Consolidación en las periferias de una estratificación social, en la que las èlites basan su predominio en su incorporación al sistema comercial extranjero.

- El cambio en la estructura económica de las periferias donde se impone la intensificación de la explotación para atender a la nueva demanda, en situaciones tipicamente colonialistas de esquilmación y agotamiento futuro de los recursos.

- La dependencia mutua entre comerciantes e indigenas, generada a partir del sistema de oferta y demanda fuertemente establecido entre ellos.

- La incorporación gradual de las economías periféricas a una economía de mercado premonetal, con las repercusiones de indole económica, politica y social que ello conlleva, pero que, por su amplitud, merecen el espacio de otro trabajo.

\section{BIBLIOGRAFIA}

Appadural, A. (1986): «Introduction: commodities and the politics of value", en A. Appadurai, (Ed.): The Social Life of Things: commodities in cultural perspective. Cambridge, 3-63.

Auвет, M. E. (1984): «La aristocracia tartésica durante el periodo Orientalizante», en Opus III, 445468.

Auвет, M. E. (1987): Tiro y las colonias fenicias de Occidente. Barcelona, Ed. Bellaterra.

AuBet, M. E. (Coord.) (1989), Tartessos, Arqueologia protohistórica del Bajo Guadalquivir. Barcelona, Ed. Ausa.

Bakmuizen, S. C. (1976): Chalcis-in-Euboea, Iron and Chalcidians Abroad, Chalcidian Studies III, Leiden.

BERARD, C. (1970): L'Hérôon à la porte de l'ouest. Eretria. Berna.

BernardinI, P. (1988): «S,Antioco: Area del Cronicario (Campagne di scavo 1983-86). Línsediamiento fenicion, en R.S.F. XVI, 1, 75-89.

BERNARDINI, P. (1991): «Un insediamento fenicio a Sulci nella seconda metá dell'VIII sec .a.C.», en Atti II Congr. Intern. Studi Fenici e Punici (1987), Roma, 665-673.

BISI, A. M. (1983): «Importazioni e imitazioni greco-geometriche nella più antica ceramica fenicia d'Occidente». Atti I Congresso Internazionale di Studi Fenici e Punici. Roma, 693-716. 
BRUMF 1:1., E. M., y EARI1. T. K. (1987): «Specialization, exchange and complex societies: an introduction", en E. M. Brumfiel, y T. K. Earle, (Eds.): Specialization, exchange and complex societies, Cambridge Univ. Press, 1-9.

BüchNi:R, G. (1982): "Die Beziehungen zwischen der euboïschen Kolonie Pithekoussai auf der Insel Ischia und dem nordwestsemitischen Mittelmeerraum in der zweiten Hälfte des 8. Jhs.v.Chr.», en Niemeyer, H. G., Phönizier im Hesten, Madrider Beiträge 8, 277-297.

Cabrira, P. (1986): «Los griegos en Huelva: los materiales griegos", en Homenaje a Luis Siret, Cuevas del Almanzora, Almería (1984). Sevilla.

Cabrera, P. (1988-89): «El comercio foceo en Huelva: cronologia y fisionomia», en Fernández Jurado, J.: Tartessos y Huelva, Huelva Arqueológica $\mathrm{X}-\mathrm{XI}, 43-100$.

CABRI:RA, P. (en prensa): «Importaciones griegas arcaicas en el Cerro del Villar (Guadalhorce, Málaga)", en Cabrera, P.; R. Olmos, y E. Sanmarti, (Coord.): Simp. Int. Griegos e Iheros, Ampurias. 1991. Huelva Arqueológica.

CabrerA, P., y Olmos, R. (1985): «Die Griechen in Huelva. Zum Stand der Diskussion», en Madrider Mitteilungen 26, 61-74.

Coldotran, J. N. (1968): Greek Geometric Potterv. Londres.

Coldstriam, J. N. (1977): Geometric Greece. Londres.

Coldstream, J. N. (1982a): «Some Problems of Eighth Century Pottery in the West seen from the Greek Angle», en La céramique grecque ou de tradition grecque au VIII siècle en Italie Centrale et Méridionale. Cahiers du Centre J.Berard III. $21-38$

Coldstream, J. N. (1982b): «Greeks and Phoenicians in the Aegean», en H. G. Niemeyer, Phönizier im Westen, Madrider Beiträge 8, 261-273.

Champion, T. C. (Ed.) (1989): Centre and Periphery. Comparative Studies in Archaeology. Londres, Unwin Hyman.

D'Agostino, B. (1985): «La formazione dei centri urbani», en M. Cristofani (Ed.): Civiltá degli etruschi. Milan, 43-47.

FERNÁNDEZ JURADO, J. (1989): «La orientalización de Huelva», en M. E. Aubet, (Coord.), Tartessos, Arqueología protohistórica del Bajo Guadalquivir. Barcelona, Ed. Ausa. 209-243.

FINLEY, M. (1973): The Ancient Economy. Londres.

Frankenstein, S. (1979): "The Phoenicians in the Far West: a function of Neo-Assyrian imperialism", en M. T. Larsen (Ed.): Power and Propaganda. Copenhague.
FravkI NSIH S., y Row1 MNハ, M. (1978): «The internal structure and regional context of Early Iron Age society in southwest (iermany", en Bull. Inst. Arch. of London 15, 73-112.

HASE BR() K. J. (1965): Trade and Politics in Ancient Grece. Nueva York.

JimINTON, A. W. y JoNIS, R. E. (1978): «The S.O.S Amphoran, B.S.A. 73, 103-141.

K、APP, B. (1993): "Thalassocracies in Bronze Age eastern Mediterranean Trade: making and breaking a myth", en World Archaeology 24,3, 332347.

KopytoH, I. (1986): "The Cultural Biography of Things: commodization as process», en A. Appadurai, (Ed.): The Social Life of Things: commodities in cultural perspective. Cambridge, 64-91.

MARTELli, M. (1981): «Populonia: cultura locale e contatti con il mondo greco", en L'Etruria Mineraria. Atti XII Conv. Studi Etruschi, (1979), 399-427.

MARTI:LL.1, M. (1991): «I Fenici e la questione orientalizzante in Italia», en Atti II Congr. Intern. Studi Fenicie Punici (1987), Roma, 1049-1072.

Mc Cadams, R. (1974): «Anthropological Perspectives on Ancient Trade", en Current Anthropo$\log _{v} 15,3,239-258$

MelAS, M. (1991): «Mediterranean Trade in the Bronze Age: A Theoretical Perspective», en N. H. Gale, (Ed): Bronze Age Trade in the Mediterranean, Studies in Mediterranean Archaeology, XC, 387 398.

Nifmeyer, H. G. (1984): «Die Phönizer und die Mittelmeerwelt im Zeitalter Homers», en RGZM 31 , $1-96$.

Pellicer, M. (1962): «Excavaciones en la necrópolis púnica 'Laurita' del Cerro de San Cristóbal (Almuñecar, Granada)", en Excavaciones Arq. en España 17.

RenFrew, C. (1969): «Trade and Culture Process in European Prehistory", en Current Anthropology 10,2-3, 151-160.

RENFREW, C. (1984): Approaches to Social Archaeo$\log y$, Harvard Univ. Press.

RIDGWAY, D. (1982): «The eighth century Pottery at Pithekoussai: an interim report». La céramique grecque ou de tradition grecque au VIII siècle en Italie Centrale et Méridionale. Cahiers du Centre J. Bérard III. 69-102.

Rızzo, M. A. (1990): Le anfore da transporto e il commercio etrusco arcaico I. Studi di Archeologia 3. Sopra.Arch. per L'Etruria Meridionale.

Rızzo, M. A. (1991): «Alcune importazioni fenicie da Cerveteri». Atti II Congresso Internazionale di Studi Fenici e Punici. Roma. 1169-1181. 
Rofвuck, C. (1984a): "Three ('lasses (?) in Early Attican, en C. Roebuck. (Ed.): E(onomy and Societs in the Early. Greek World. Chicago, 85-93.

Rorbuck, C. (1984b): "Some aspects of urbanization in Corinth", en C. Roebuck. (Ed.): Econom! and Societs in the Early Greek World. Chicago. 96-127.

RotnI.ARD, P. (1990): «Phéniciens et grecs à Toscanos. Note sur quelques vases d'inspiration gréco-géométrique de Toscanos (1967)", en Madrider Mitteilungen 31, 178-185.

Rowl.ANDS, M. (1987): "Centre and Periphery: a review of a concept", en M. Rowlands, M. T. Larsen, y K. Kristiansen, (Eds.): Centre and Periphery in the Ancient World, Cambridge.

Ruiz MatA, D. (1989): "Huelva: un foco temprano de actividad metalúrgica durante el Bronce $\mathrm{Fi}$ nal", en M. E. Aubet (Coord): Tartessos, Arqueologia protohistórica del Bajo Guadalquivir. Barcelona, Ed. Ausa. 209-243.

Ruiz Mata, D. (1993): "Los fenicios de época arcaica - siglos VIII/VII - en la Bahia de Cádiz. Estado de la cuestión". Instituto Oriental, Lisboa.

Schortman, E. M., y Urban, P. A. (1987): «Modeling Interregional Interaction in Prehistory», en Advances in Archaeological Method and Theory, vol. 11. Academic Press, 37-95.

Shefton, B. B. (1982): «Greeks and Greek Imports in the South of the Iberian Peninsula», en H. G. Niemeyer, Phönizier im Westen, Madrider Beiträge $8,337-370$.

Shi:Rrat, A., y S. (1991): "From Luxuries to Commodities: The Nature of Mediterranean Bronze Age Trade Systems», en N. H. Gale, (Ed): Bronze Age Trade in the Mediterranean, Studies in Mediterranean Archaeology, XC, 351-384.

Silfrrat, A.. y S. (1993): "The growth of the Mediterranean economy in the early first millenium $\mathrm{BC}$, en World Archaeology 24.3, 361-377.

Sombart, W. (1967): Luxury and Capitalism. Ann Arbor.

StadDARt, S. (1989): «Divergent trajectories in central Italy, 1200-500 B. C.», en T. C. Champion, (ED.), Centre and Periphery. Comparative Studies in Archaeologv, Londres, 88-101.

Strom, I (1991): "Il ruolo del commercio fenicio del Villanoviano in Etruria. Un'ipotesin, en Atti II Congresso Internazionale di Studi Fenici e Punici. Roma. 323-331.

VEGiAs, M. (1989): «Archaische und mittelpunische Keramik aus Karthago», en Roemische Mitteilungen 96, 209-265.

WALLERSTEIN, I. (1974): The Modern World-System: Capitalist Agriculture and the Origins of the European World-Economy in the Sixtheen Century. Nueva York. 